\title{
Observation of Direct Ion Heating in Double-Pulsing CHI in Helicity Injected Spherical Torus Plasmas
}

\author{
Takafumi HANAO, Hidetoshi HIRONO, Takahiro HYOBU, Kengo ITO, Keisuke MATSUMOTO, \\ Takashi NAKAYAMA, Yusuke KIKUCHI, Naoyuki FUKUMOTO and Masayoshi NAGATA \\ Graduate School of Engineering, University of Hyogo, Hyogo 671-2280, Japan
}

(Received 18 April 2013 / Accepted 24 May 2013)

\begin{abstract}
Anomalous ion heating has been observed for the first time during flux/current amplification by doublepulsing coaxial helicity injection (CHI) in the helicity injected spherical torus (HIST) device. Doppler ion temperature increases significantly near the separatrix on the inboard side during the second CHI pulse, whereas electron temperature remains constant. The experimental results indicate that selective ion heating may be associated with viscous damping of poloidal flows driven by the $\mathrm{CHI}$ pulse.
\end{abstract}

(c) 2013 The Japan Society of Plasma Science and Nuclear Fusion Research

Keywords: coaxial helicity injection, anomalous ion heating, spherical torus, viscous flow damping, current amplification

DOI: $10.1585 /$ pfr.8.1202104

Coaxial Helicity Injection (CHI) using a magnetized coaxial plasma gun (MCPG) is a useful method of noninductive plasma start-up and dynamo current drive for spherical torus (ST) plasmas. The new CHI operation called "Multi-pulsing" proposed as an approach to good time-averaged confinement was successfully demonstrated for the SSPX spheromak device [1]. Multi-pulsing operation of ST devices has been applied for the first time to the HIST device. Double-pulsing CHI operation has successfully increased the sustainment time beyond that obtained with single $\mathrm{CHI}[2]$.

The ion temperature $T_{\mathrm{i}}$ is normally observed to be comparable to the electron temperature $T_{\mathrm{e}}$ in Ohmic tokamak plasmas. The externally input energy is predominantly dissipated by the current, leading to electron heating. Ions are then normally heated by frictional drag through collisions with electrons. Anomalous ion heating $\left(T_{\mathrm{i}}>T_{\mathrm{e}}\right)$ has been observed in several experiments, but its mechanism is not fully understood. For example, such ion heating was observed similarly during a sawtooth crash in the MST-RFP device [3] and during a plasma merging process in the TS-3/TS-4 devices [4]. The anomalous ion heating is thought to be attributed to viscous damping of the flow associated with tearing modes, magnetohydrodynamic relaxations, and magnetic reconnections. In this article, we report the first observation of ion heating during current amplification in double-pulsing CHI experiment in the HIST. The structures, sizes, capabilities, diagnostics, and operating conditions of the HIST are described in detail in Ref. [5]. The Doppler spectroscopy diagnostic for the ion temperature $T_{\mathrm{i} . \mathrm{D}}$ is described briefly here. The Ion Doppler Spectrometer (IDS) system has a compact 16-channel photomultiplier tube, optical fibers, and a

author's e-mail: eul3s003@steng.u-hyogo.ac.jp

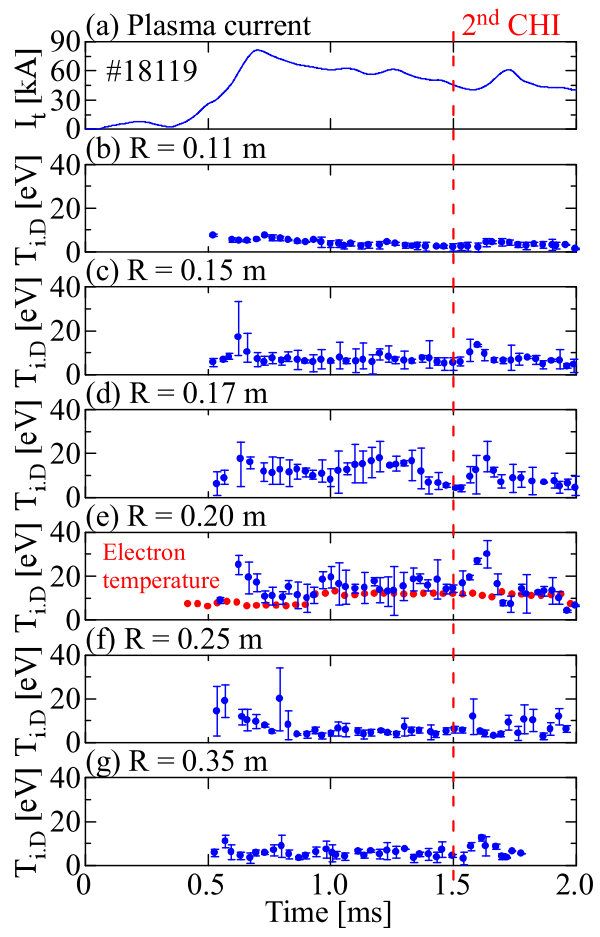

Fig. 1 Time evolution of (a) $I_{\mathrm{t}}$ and (b) $-(\mathrm{g}) T_{\mathrm{i} . \mathrm{D}}$ at each radial position. The electron temperature $T_{\mathrm{e}}$ is plotted at (e) $R=$ $0.20 \mathrm{~m}$. The second CHI pulse is applied at $t=1.5 \mathrm{~ms}$.

$1-\mathrm{m}$-spectrometer (total system resolution: $0.031 \mathrm{~nm}$ ). The technical details, including the spatial resolution of a similar IDS system, are reported in Ref. [6]. In this experiment, an optical fiber covered with a glass tube is inserted into the plasma to measure the radial profiles of the Doppler ion temperature $T_{\text {i.D }}$. The optical fiber collects the light emitted from the plasma through a quartz window with a 
mirror installed at the head of the glass tube. The viewing chord is on the poloidal cross section. The optical fiber is scanned radially at intervals of $0.05 \mathrm{~m}$ shot by shot. The IDS measured $T_{\text {i.D }}$ using the OII impurity spectral line $(441.49 \mathrm{~nm})$. The electron temperature was measured using a double electrostatic probe. A triangular waveform voltage with a sweep frequency of $25 \mathrm{kHz}$ was applied between the double probe electrodes. The radial electric field $E_{\mathrm{r}}$ was measured by radially scanning the double probe on the midplane of the flux conserver.

In this experiment, the second sustainment capacitor bank is triggered at $t=1.5 \mathrm{~ms}$ during the decay phase of the initial plasma. Figure 1 shows the time evolution of the toroidal current $I_{\mathrm{t}}$ and $T_{\mathrm{i} . \mathrm{D}}$ at each radius. The second $\mathrm{CHI}$ pulse amplified $I_{\mathrm{t}}$ effectively against the resistive decay and extended the life-time $t_{\text {life }}$ to $\sim 4 \mathrm{~ms}$, which is longer than that with single CHI. The time period for which the second CHI effectively provides current amplification is about $0.3 \mathrm{~ms}$. The magnetic axis and separatrix of the initially formed ST configuration are located at $R \sim 0.25 \mathrm{~m}$ and $R \sim 0.15 \mathrm{~m}$, respectively. During the first CHI start-up phase ( $t=0.5-0.7 \mathrm{~ms}$ ), $T_{\mathrm{i} . \mathrm{D}}$ at $R=0.2 \mathrm{~m}$ is higher than $T_{\mathrm{e}}$, as shown in Fig. 1 (e). After the second CHI pulse, $T_{\text {i.D }}$ started to increase rapidly again and reached a peak value of $30 \mathrm{eV}$ at $R=0.20 \mathrm{~m}$. The increase in $T_{\mathrm{i} . \mathrm{D}}$ is correlated with that in $I_{\mathrm{t}}$, whereas the electron temperature, $T_{\mathrm{e}} \sim 10 \mathrm{eV}$, remains unchanged, as shown in Fig. 1 (e). This observation of $T_{\mathrm{i} . \mathrm{D}}>T_{\mathrm{e}}$ indicates direct ion heating, and this behavior is similar to the characteristics of reconnection events $[3,4]$. After current amplification was completed, $T_{\text {i.D }}$ cooled to the original level.

Figures 2 (a) and (b) show changes in the radial profiles of $T_{\mathrm{i} . \mathrm{D}}$ and $T_{\mathrm{e}}$ during the second CHI pulse. The radial profiles of $T_{\text {i.D }}$ peak at $R=0.20 \mathrm{~m}$. The electron temperature $T_{\mathrm{e}}$ is almost uniform in the closed flux region, whereas in the open flux column (OFC) region ( $0.06 \mathrm{~m}<R<0.15 \mathrm{~m}), T_{\mathrm{e}}$ is higher. Ion heating occurs mostly in the region between $R=0.15 \mathrm{~m}$ and $R=0.20 \mathrm{~m}$, where the direction of the poloidal flow is reversed at $R \sim 0.17 \mathrm{~m}$, so the flow shear is largest [2]. In the OFC region, ion heating does not appear because of the strong toroidal magnetic field. Figure 2 (c) indicates that during the second CHI pulse, the polarity of $E_{\mathrm{r}}$ in the OFC region of $R=0.10-0.15 \mathrm{~m}$ is positive, whereas it is negative in the closed flux region. This self-generated radial profile of $E_{\mathrm{r}}$ at $t=1.49 \mathrm{~ms}$ may be attributed to non-ambipolarity diffusion toward the plasma core [7]. The positive $E_{\mathrm{r}}$ is oriented to the core region. When the second $\mathrm{CHI}$ pulse is applied between the electrodes (the center conductor is biased to be negative), $E_{\mathrm{r}}$ changes the polarity in the region

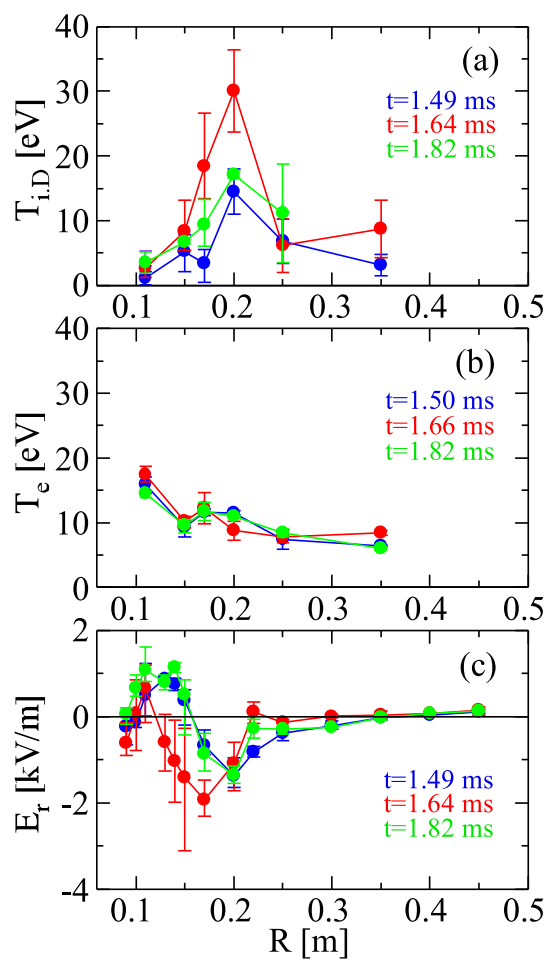

Fig. 2 Radial profiles of (a) $T_{\text {i.D }}$, (b) $T_{\mathrm{e}}$, and (c) $E_{\mathrm{r}}$ during the second CHI pulse.

of $R=0.12-0.15 \mathrm{~m}$, and its negative value at $R=0.17 \mathrm{~m}$ increases from $-0.8 \mathrm{kV} / \mathrm{m}$ to $-2 \mathrm{kV} / \mathrm{m}$. This significant change in the polarity and amplitude of $E_{\mathrm{r}}$ accelerates the poloidal flow in the opposite direction owing to $\boldsymbol{E} \times \boldsymbol{B}$ drift. Poloidal shear flow is observed in this region which may be caused by the combined effects of $\boldsymbol{E} \times \boldsymbol{B}$ drift and the diamagnetic drift of ions [2]. The second CHI pulse enhances the poloidal flow there by $\boldsymbol{E} \times \boldsymbol{B}$ drift. Consequently, the acceleration of this perpendicular flow in the region is thought to produce direct heating of ions through viscous flow damping.

This study was supported by a Grant-in-Aid for Scientific Research (No.22360391) from the Japan Society for the Promotion of Science.

[1] B. Hudson et al., Phys. Plasmas 15, 056112 (2008).

[2] M. Nagata et al., 24th IAEA Fusion Energy Conference, ICC/1-1Rb (2012).

[3] E. Scime et al., Phys. Rev. Lett. 68, 2165 (1992).

[4] Y. Ono et al., Phys. Plasmas 4, 1953 (1997).

[5] M. Nagata et al., Phys. Plasmas 10, 2932 (2003).

[6] P. Gu et al., Rev. Sci. Instrum. 75, 1337 (2004).

[7] K. Ida, Plasma Phys. Control. Fusion 40, 1429 (1998). 